\title{
Mast cell synapses and exosomes: membrane contacts for information exchange
}

\author{
Amanda Carroll-Portillo ${ }^{1}$, Zurab Surviladze ${ }^{1}$, Alessandra Cambi ${ }^{2}$, Diane S. Lidke ${ }^{1}$ and Bridget S. Wilson ${ }^{1}$ * \\ 1 Department of Pathology, University of New Mexico Health Sciences Center, Albuquerque, NM, USA \\ ${ }^{2}$ Department of Tumor Immunology Nijmegen, Nijmegen Centre for Molecular Life Sciences, Radboud University Nijmegen Medical Centre, Nijmegen, \\ Netherlands
}

\section{Edited by:}

Toshiaki Kawakami, La Jolla Institute

for Allergy and Immunology, USA

\section{Reviewed by:}

Carlo Pucillo, University of Udine, Italy Yoseph A. Mekori, Meir Medical Center and Tel Aviv University, Israel Francesca Levi-Schaffer, The Hebrew University of Jerusalem, Israel Kenji Kabashima, Kyoto University, Japan

\section{${ }^{*}$ Correspondence:}

Bridget S. Wilson, Department of

Pathology, University of New Mexico Health Sciences Center, MSC08

4640, CRF205, 1 University of New

Mexico, Albuquerque, NM 87131,

USA.

e-mail:bwilson@salud.unm.edu
In addition to their central role in allergy, mast cells are involved in a wide variety of cellular interactions during homeostasis and disease. In this review, we discuss the ability of mast cells to extend their mechanisms for intercellular communication beyond the release of soluble mediators. These include formation of mast cell synapses on antigen presenting surfaces, as well as cell-cell contacts with dendritic cells and T cells. Release of membrane bound exosomes also provide for the transfer of antigen, mast cell proteins, and RNA to other leukocytes. With the recognition of the extended role mast cells have during immune modulation, further investigation of the processes in which mast cells are involved is necessary. This reopens mast cell research to exciting possibilities, demonstrating it to be an immunological frontier.

\section{Keywords: mast cell, synapse, exosome, dendritic cell}

\section{INTRODUCTION}

Mast cell IgE-driven responses are critical to immunity against invading parasites. However, in developed countries where parasitic infections are minimal, these cells have become central to allergic responses. Thus allergy-related issues have been the primary focus of mast cell research for several decades. The central player in most studies has been the high-affinity IgE receptor, Fc\&RI. Following antigen-mediated crosslinking, the Fc\&RI signaling pathway stimulates secretion of mast cell soluble factors (i.e., histamine, leukotrienes, prostaglandins, and cytokines) responsible for allergic symptoms.

In the last decade, the immunological role of mast cells has been steadily expanding. Mast cells regulate other immune cells via the wide variety of cytokines and chemokines secreted upon activation (Galli and Wershil, 1995; Bachelet and Levi-Schaffer, 2007). Additionally, mast cells express receptors that mediate innate immunity, such as toll-like receptors (TLRs; Supajatura et al., 2001; Matsushima et al., 2004), leading to the hypothesis that mast cells can play a significant role in immune responses to infection and other environmental challenges. This hypothesis is strongly supported by evidence that mast cells influence the behavior of $\mathrm{T}$ cells (Hershko and Rivera, 2010; Valitutti and Espinosa, 2010) B cells (Merluzzi et al., 2010), dendritic cells (Shelburne et al., 2009; Dawicki et al., 2010), macrophages (Ketavarapu et al., 2008), neurons (Buhner and Schemann, 2012), and even tumor cells (Ribatti and Crivellato, 2011). Importantly, mast cells and other immune cells interact in complex ways that include both soluble mediators and direct contact. Here, we focus specifically on the mechanisms by which mast cells communicate with other hematopoietic cells via cell-cell contacts ("synapses") and delivery of mast cell cargo through binding and uptake of membrane-derived vesicles ("exosomes"). The rapidly accelerating pace of the field suggests that we have just begun to appreciate the importance of mast cells in the immune system, and that this is indeed an exciting frontier in inflammatory research.

\section{MAST CELL SYNAPSES}

One way in which immune cells communicate to modulate systemic responses is through direct contact, such as that seen with formation of the immunological synapse (Thery et al., 2009; Dustin et al., 2010). Mast cells participate in mast-T cell synapse formation as antigen presenting cells, which is possible through mast cell display of peptide-loaded MHCII and co-stimulatory molecules (Bhattacharyya et al., 1998; Valitutti and Espinosa, 2010). MHCII surface expression by primary mouse mast cells (bone marrow and peritoneal derived mast cells; BMMCs, PCMC) and human mast cell lines requires stimulation with IFN $\gamma$ or IL-4 treatment during growth (Grabbe et al., 1997; Gaudenzio et al., 2009; Valitutti and Espinosa, 2010). In this context, mast cells and $\mathrm{T}$ cells can form a "traditional" immunologic synapse that mediates antigen-specific $\mathrm{T}$ cell activation. It is reasonable to speculate that this may be an important feature in establishing and maintaining the atopic state, since one important outcome of IgE-mediated crosslinking is the endocytosis of antigen (Pfeiffer et al., 1985). Once delivered to secretory lysosomal compartments, allergens are subjected to proteolysis, and peptides are generated with potential for loading onto MHCII. 
We considered the possibility that mast cells could also be on the "receiving" end of a synapse, if presented with antigen on the surface of another cell. Like the $\mathrm{T}$ cell and $\mathrm{B}$ cell receptors (TCR, BCR), FceRI is a multisubunit immuno-receptor that utilizes ITAM motifs to initiate a tyrosine kinase signaling cascade (Tamir and Cambier, 1998). As initial test of this possibility, we presented IgE-primed mast cells with a planar, lipid bilayer containing mobile, cognate, monovalent ligand. This represents a modification of the technique first developed by Dustin et al. (1996) to characterize the $\mathrm{T}$ cell synapse.

We were able to confirm that a number of mast cells (including $\mathrm{MC} / 9, \mathrm{RBL}-2 \mathrm{H} 3$ cell lines, and primary BMMCs) readily form FceRI-centric synapses upon recognition of antigen within bilayers (Carroll-Portillo et al., 2010; Figure 1, from Lidke and Wilson Labs). These synapses are remarkably similar to those seen with $\mathrm{T}$ cells and B cells (Carrasco et al., 2004; Dustin, 2008), suggesting that the mechanisms for synapse formation are shared among most leukocyte lineages. As mast cells primed with DNP-specific IgE settle onto a fluid bilayer containing monovalent DNP-lipid, FceRIIgE complexes at the contact points bind the cognate ligand within the bilayer surface. As synapse development progresses, additional FceRI-IgE accumulate at the centralized portion of the contacting membrane (Figure 1A). Like T and B cells (Lin et al., 2008; Shefler et al., 2010), the actin cytoskeleton undergoes rearrangement to concentrate at the cell periphery, and becomes largely excluded from the central regions containing engaged receptors (Figure 1B). Cholesterol also concentrates within the contacting membrane where engaged FceRI-IgE reside (Figure 1C); in conjunction with the loss of underlying actin cytoskeleton, this allows for unconfined mobility of engaged receptors. Furthermore, only bound FceRI-IgE segregates into the fluid region of the contacting membrane as demonstrated by failure of IgE specific for a second antigen to partition there (Figure 1D).

While soluble monovalent antigen is insufficient to stimulate degranulation from mast cells (Pfeiffer et al., 1985), presentation of monovalent ligand within the bilayer results in a low, but statistically significant level of degranulation (Carroll-Portillo et al., 2010). This result is best explained by the observation that up to $3 \times 10^{5}$ IgE receptors accumulate at the contacting membrane. This high density markedly increases the rate of encounters between IgE-FceRI complexes. Since the receptors remain highly mobile, these monovalent interactions must be extremely transient but collectively generate sufficient kinase activation to propagate measureable stimulatory signals.

Pharmacologic studies further established the contributions of actin and Lyn/Fyn kinase activity to mast cell synapse organization. Treatment of IgE-primed mast cells with the actin-disrupting drug Latrunculin B prior to addition to the DNP-lipid bilayer resulted in disruption of synapse architecture (Figure 1E, upper row). In contrast, mast cells treated with PP2 (a Src family kinase inhibitor that targets the mast cell kinases Lyn and Fyn) could form synapses, but the cytoskeleton reorganization was incomplete (Figure 1E, lower row). These observations support the conclusion that actin remodeling at the synapse is downstream of local kinase activation.

Do mast cell synapses of this type occur in vivo? We expect so, especially in the context of live parasites and bacteria whose cell membranes are decorated with antigenic epitopes. Early electron microscopy evidence demonstrated tight binding between rat peritoneal mast cells and Schistosoma mansoli larvae (Caulfield et al., 1981) and BMMCs degranulate when incubated with live Leishmania (Bidri et al., 1997). Antibody-mediated direct contact can also lead to mast cell phagocytosis of the parasite (Saha et al., 2004). An important outstanding question is whether other mast cell receptors implicated in direct binding to pathogens, such as the TLRs and the mannose-binding protein CD48 (Marshall, 2004) also reorganize into synapse-like structures at the point of contact.

There is also strong evidence that contact of mast cells with activated $T$ cells can lead to mast cell degranulation and cytokine production (Bhattacharyya et al., 1998). This is dependent upon integrin (LFA-1) and ICAM interactions between the adherent cells (Inamura et al., 1998), and can occur in the context of mast cell antigen presentation (Gaudenzio et al., 2009). Thus mast cell-T cell synapses can lead to bi-directional signaling, creating a positive feedback situation expected to drive inflammatory responses in tissues (Mekori and Baram, 2002).

\section{THE SPECIAL CASE OF MAST CELL-DENDRITIC CELL CONTACTS}

In addition to $\mathrm{T}$ cells and $\mathrm{B}$ cells, mast cells also affect the function and trafficking of dendritic cells. Dendritic cells and mast cells are both ubiquitous in tissue at the environmental interfaces, and both can affect immune responses after detection of invading pathogens (Gurish and Austen, 2001; Soloff and BarrattBoyes, 2010; Wesolowski and Paumet, 2011). Importantly, these cells are in close proximity allowing for response by dendritic cells to mast cell soluble mediators and the formation of direct contacts (Figure 2A, from Cambi and Lidke Labs). Thus, mast cells and dendritic cells are potential partners in modulation of immune responses to environmental changes.

Dendritic cells have long been considered the sentinels of the immune system (Cella et al., 1997; Banchereau and Steinman, 1998). They reside in an immature state within the tissue and work in this environment to sample antigens through phagocytosis. Upon stimulation with inflammatory cytokines or recognition of a non-self antigen, dendritic cells begin trafficking through the tissue to the draining lymph node. Several maturation markers become upregulated (such as CD80, CD40, CD86, MHCII, and CCR7), and antigen is presented to lymphocyte populations within the lymph node to stimulate proliferation, thus initiating the immune response.

Mast cells secrete a remarkable repertoire of soluble mediators, including histamine, leukotrienes, prostaglandins, cytokines, and chemokines. Close proximity of mast cells within the tissue, as well as the numerous cytokine/chemokine receptors expressed by dendritic cells, allows for response to these soluble mediators upon mast cell activation. Supernatant from activated mast cells has been shown to induce upregulation of dendritic cell maturation markers in mouse and human systems (Caron et al., 2001; Kitawaki et al., 2006), increase CCL21 chemotaxis to the draining lymph nodes (Shelburne et al., 2009; Ren et al., 2010), and stimulate Th2-promoting dendritic cells (Kitawaki et al., 2006). Specifically, histamine and $\mathrm{TNF} \alpha$ play large roles in these changes. $\mathrm{TNF} \alpha$ works locally to increase the expression of E-cadherin that, in conjunction with other inflammatory cytokines, serves 
A

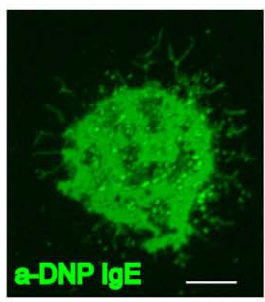

B

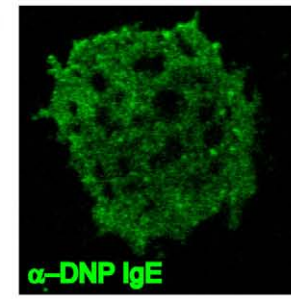

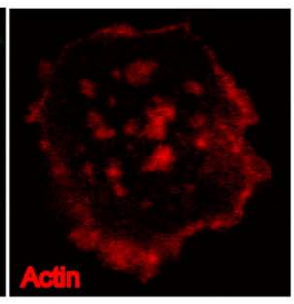

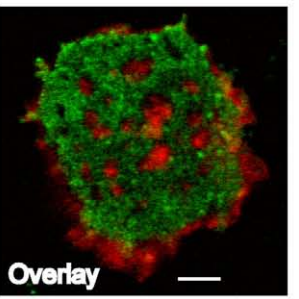

C
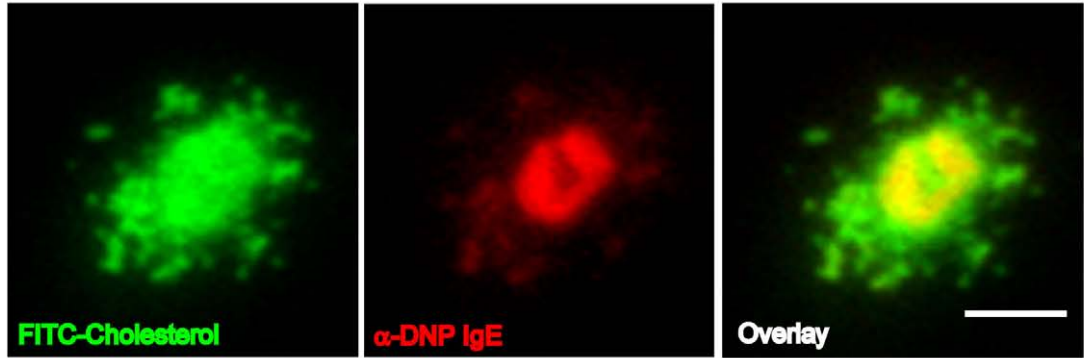

D

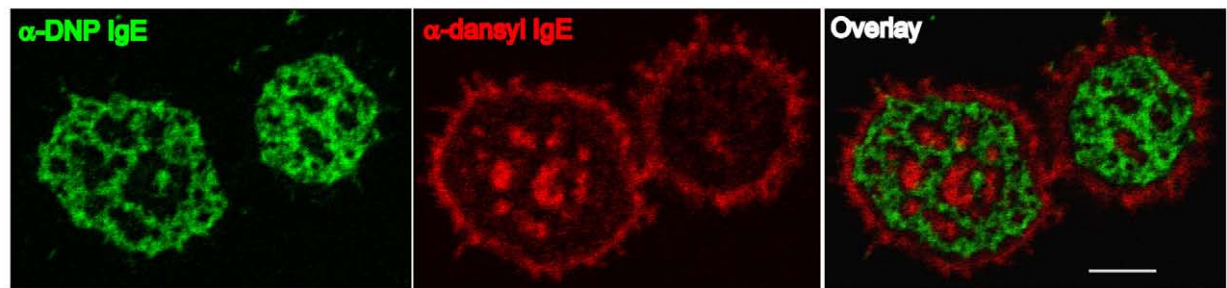

$\mathbf{E}$
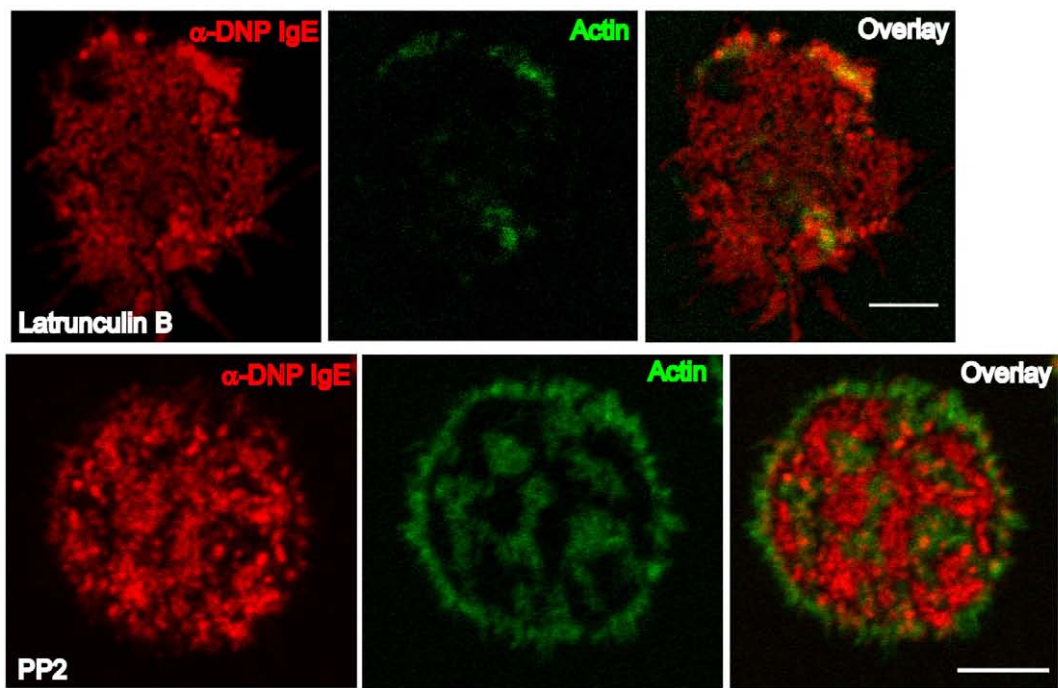

FIGURE 1 | Characteristics of the mast cell synapse. (A) Confocal section demonstrating synapse formed by a MC/9 mast cell primed with $\alpha$-DNP $\lg \mathrm{E}_{\mathrm{A} 488}$ after $20 \mathrm{~min}$ on a $25 \mathrm{~mol} \%$ DNP-lipid bilayer. (B) Structure of the actin cytoskeleton in a RBL cell primed with $\alpha$-DNP $\lg \mathrm{E}_{\mathrm{A} 488}$ after 20 min on a 25 mol\% DNP-lipid bilayer. Confocal section of cells fixed with paraformaldehyde and then labeled with rhodamine phalloidin to visualize actin. (C) Total internal reflection fluorescence microscopy (TIRFm) image of an RBL cell repleted with FITC-conjugated cholesterol and primed with $\alpha$-DNP
IgE $\mathrm{E}_{\mathrm{A} 555}$ after $20 \mathrm{~min}$ on a $25 \mathrm{~mol} \%$ DNP-lipid bilayer. (D) Confocal section of RBLs co-primed with equal concentrations of $\alpha$-DNP Ig $E_{A 488}$ and $\alpha$-dansyl $\lg E_{A 555}$ and settled onto $25 \mathrm{~mol} \%$ DNP-lipid bilayers after $20 \mathrm{~min}$. (E) Confocal sections of RBLs primed with $\alpha$-DNP $\lg _{A 555}$ and pretreated with either Latrunculin B (upper row) or PP2 (lower row) settled on 25 mol\% DNP-lipid bilayers for $20 \mathrm{~min}$. Cells were paraformaldehyde fixed and stained with A488 phalloidin. All images have been brightness and contrast enhanced. Scale bars are $5 \mu \mathrm{m}$ to recruit dendritic cells to the site of infection (Shelburne et al., 2009). Peripherally, TNF $\alpha$ stimulates changes within the lymph node environment causing enlargement (Kunder et al., 2009) and increased expression of CCL21 with subsequent upregulation of dendritic cell chemotaxis (Shelburne et al., 2009; Ren et al., 2010). Histamine also locally stimulates upregulation of dendritic cell 


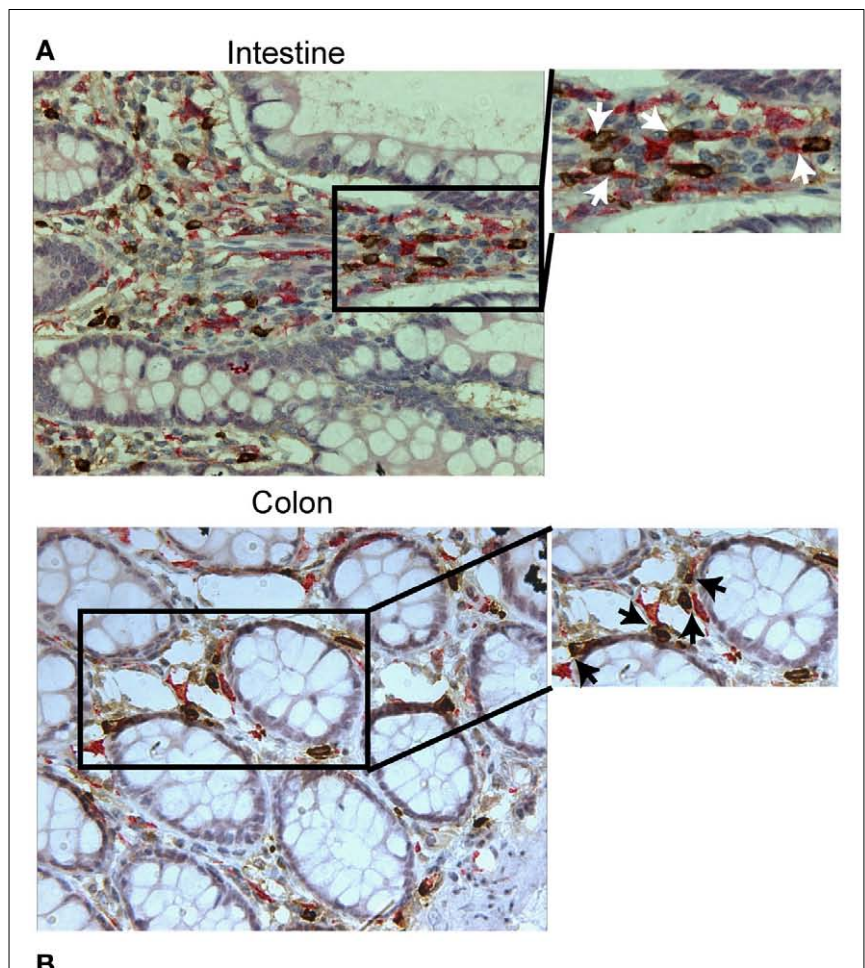

B

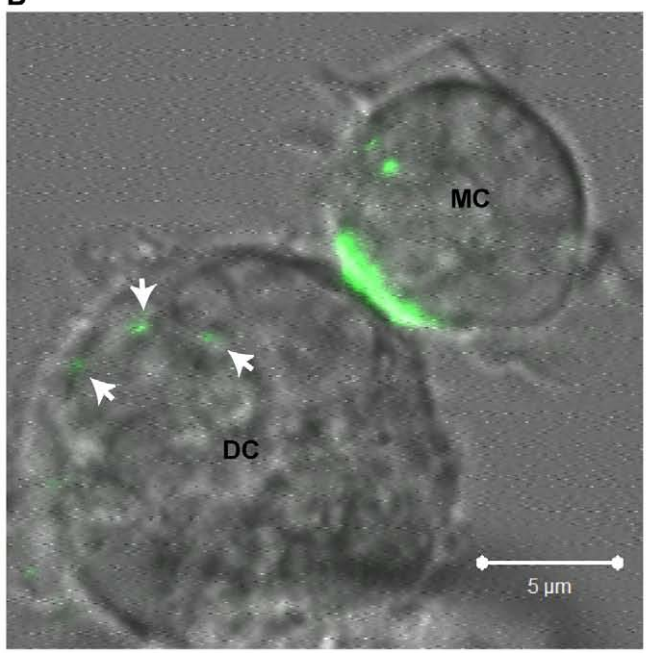

FIGURE 2 | Interactions between mast cells and dendritic cells (A) Immunohistochemistry of normal human intestinal and colon tissue double stained for mast cell tryptase (brown) and CD209 (DC-SIGN, pink). Arrows within blown up sections indicate regions where direct contact between the two cell types is observed. (B) Live cell, confocal imaging of a mouse bone marrow-derived mast cell primed with $\alpha$-DNP $\lg E_{A 488}$ (green) directly interacting with a mouse bone marrow-derived dendritic cell after activation with DNP-BSA. Arrows indicate where mast cell internalized material has been transferred to the dendritic cell. All images have been brightness and contrast enhanced.

maturation markers and expression of Th2-associated cytokines, even in the presence of LPS and IFN $\gamma$ that on their own would drive a Th1-promoting dendritic cell response (Caron et al., 2001; Kitawaki et al., 2006). Histamine increases dendritic cell capacity to stimulate proliferation of memory $\mathrm{T}$ cell populations (Caron et al., 2001), and results in the peripheral recruitment of specific dendritic cell to the draining lymph node (Dawicki et al., 2010). All of the previous examples represent interactions in response to inflammatory signals. However, mast cells also regulate dendritic cell behavior in the absence of inflammation, as seen in the instance of allograft tolerance (de Vries et al., 2011). In contrast to dendritic cell trafficking in response to inflammatory signals, GM-CSF (most likely produced by the local mast cell population) within the grafted area represses mast cell degranulation (e.g., inflammation) and increases the longevity of the immature dendritic cell population. This, in combination with mast cell-secreted $\mathrm{TNF} \alpha$, results in increased trafficking of immature dendritic cells to the draining lymph node and serves to stimulate allograft tolerance in the absence of $\mathrm{T}$ cell proliferation. Mast cells are indispensable for mediation of dendritic cell tolerance of allografts demonstrating another example of intercellular communication through soluble factors.

Although soluble interactions between mast cells and dendritic cells are well characterized, the effects of direct contact are just starting to be investigated in the context of both homeostasis (Dudeck et al., 2011) and disease (Kitawaki et al., 2006; Bacci et al., 2010; Otsuka et al., 2011). The consequences of direct interaction between activated mast cells and dendritic cells are similar to those seen with soluble mediators. Thus, direct contact can lead to upregulation of maturation markers, increased expression of Th2-specific cytokines, increased dendritic cell chemotaxis to the lymph node, and enhanced $\mathrm{T}$ cell stimulation (Kitawaki et al., 2006; Otsuka et al., 2011). It is possible that direct contact in these instances stimulates dendritic cells when only a few mast cells become activated within the tissue. Contact would allow for delivery of more concentrated doses of soluble mediators, as well as stimulating a small population of dendritic cells to respond to minimal stimulus to prime the immune system. This hypothesis is supported by the physiological changes that occur within FceRI-activated mast cells upon contact with dendritic cells. Internalized receptor complexes (with antigen bound) become polarized within the mast cell with subsequent transfer of internalized material to dendritic cells (Figure 2B, from Cambi and Lidke Labs). Material transferred could become processed and presented to lymphocyte populations within the lymph node. Dudeck et al. (2011) demonstrate that activation of mast cells through FceRI is not required for dendritic cell stimulation as interaction between peritoneal-derived mast cells and dendritic cells also results in slight, but significant upregulation of dendritic cell maturation markers, secretion of certain Th1 and Th17 polarizing cytokines, and proliferation of the $\mathrm{CD} 4^{+} \mathrm{T}$ cell population. These changes become enhanced with the addition of LPS and occur in the absence of mast cell degranulation, demonstrating a non-FcERI related role for mast cells in immune modulation.

So far, in vitro interactions between mast cells and dendritic cells have been addressed using both primary cell systems as well as established cell lines (for the mast cells). However, there is an added level of complexity to these interactions in vivo due to the different classes of dendritic cells (e.g., myeloid dendritic cells, plasmacytoid dendritic cells, langerhans cells, dermal dendritic cells, etc; Kaplan, 2010; Romani et al., 2010; Kushwah and $\mathrm{Hu}, 2011$ ) and mast cell subtypes (e.g., mucosal and connective 
tissue). With populations of dendritic cells originating from different precursors and both mast cells and dendritic cells from different tissues exhibiting varied receptor repertoire and activation patterns, mast cell-dendritic cell partnerships are likely tissue specific. For example, dendritic cells residing in the skin display a completely different phenotype when compared to intestinal or blood dendritic cells. Not only does this highlight the impressive plasticity of the dendritic cell population, but it also demonstrates the likelihood that cellular interactions are in part dictated by the surrounding environment. The challenge ahead is to understand the interplay between mast cells and dendritic cell subsets that may vary among different tissues.

\section{EXOSOMES}

The variety of mast cell soluble mediators secreted upon mast cell activation lead to modulation of immune cells both locally and at distal sites (Galli and Wershil, 1995). However, these diffusible messengers lack long term stability within the body. Here, we consider the evidence that mast cell-derived exosomes offer an additional mechanism for intercellular communication. These membrane bound vesicles might be expected to have greater stability in the blood and interstitial spaces, and they uniquely provide a means of material exchange between cells since their uptake by other immune cells delivers a "payload" to the recipient cell.

Exosomes originate from part of the endosomal pathway (Stoorvogel et al., 2002). They are small vesicles $(60-100 \mathrm{~nm}$ ) that reside in multi-vesicular bodies (MVBs) within cells until stimulated exocytosis releases them into the extracellular milieu. MVBs are formed when the membranes of vacuolar endosomes bud inwards and pinch off into the lumen of the vesicle; this process can result in accumulation of many small vesicles within the same endosomal compartment. It is their size as well as their biophysical and biochemical properties that serve to distinguish exosomes from other types of membrane vesicles secreted by cells (reviewed in Thery et al., 2009). Vesicles within MVBs are thought to have three potential fates: fusion with the lysosomal pathway to target proteins and lipids for degradation, long term residency within the MVBs to serve as protein storage compartments, or serving as a source of exosomes that are shed into the extracellular milieu upon fusion of the MVB with the plasma membrane. Exosomes are bounded by a classical bilayer structure with a specific lipid composition different from that of the plasma membrane, as well as lower lateral membrane diffusion, higher flip-flop rate, and a loss of phospholipid asymmetry (Laulagnier et al., 2004, 2005). These traits are thought to contribute to higher stability of the exosomes at neutral $\mathrm{pH}$, extending their lifetime in the extracellular environment, and permitting fusion with other cellular membranes.

There is an intimate relationship between the mast cell endosomal and secretory pathways, and mast cell granules are classified as modified lysosomes (i.e., "secretory lysosomes; Blott and Griffiths, 2002). Raposo et al. (1997) described three types of endosomal/lysosomal compartments in BMMCs: type I compartments which colocalize with mannose-6-phosphate receptors, lamp-1, and lamp 2, type II which colocalize with serotonin and have an electron dense core, and an electron dense type III compartment. Both type I and type II are MVBs that contain exosomes. The exosomes within the different compartments possess distinct protein profiles (Skokos et al., 2003; Figures 3A,B from the Wilson lab). The differences between exosome populations within mast cells could be indicative of an ability of mast cells to modulate the immune response differentially based on activation state. Consistent with this hypothesis, although all mast cell lines (MC/9, P815, RBL-2H3, and HMC-1) and BMMCs pretreated with IL-4 can secrete exosomes constitutively (Skokos et al., 2001a,b), secretion of exosomes specifically from the secretory MVBs/lysosomes (those that colocalize with serotonin) requires mast cell activation through FceRI crosslinking or treatment with a $\mathrm{Ca}^{2+}$ ionophore (Raposo et al., 1997; Vincent-Schneider et al., 2001).

A wide variety of cells secrete exosomes (Denzer et al., 2000), and the presence of exosomes in many biological fluids, including breast milk (Admyre et al., 2007), urine (Pisitkun et al., 2004), saliva (Palanisamy et al., 2010), plasma (Caby et al., 2005), and bronchoalveolar lavage fluid (Dawicki et al., 2010), strongly suggests a physiological role in modulation of immune responses. Although there are proteins that are typical to exosomes (e.g., tetraspanins, cytoskeletal proteins, and chaperones), the actual content varies depending on the cellular source. Thus, it is reasonable to expect that exosomes derived from different cells will lead to very specific effects on other target cells and tissues. Mast cell exosomes have been reported to contain anywhere from 200 to 400 different proteins, and although many of these proteins are not identified, known constituents include $\mathrm{TNF} \alpha$, prothrombin and Plasminogen Activator Inhibitor Type I (Al-Nedawi et al., 2005).

Considerable attention has been paid to the functional roles for MHCII on mast cell exosomes (Raposo et al., 1997; VincentSchneider et al., 2001). Raposo et al. (1997) characterized the accumulation of MHCII molecules in the prelysosomal/lysosomal compartments of BMMCs, where exosomes are located. MHCII molecules were found almost exclusively on exosome membranes, with only a small percentage of labeling occurring on the plasma membrane or the limiting membrane of the MVB. BMMCs, as well as RBL-2H3 cells transfected to express MHCII, show slow maturation of MHCII such that persistent association of the invariant chain can obscure peptide loading (Raposo et al., 1997; VincentSchneider et al., 2001). Skokos et al. (2003) suggested that even if MHCII-peptide presentation is low, antigen can be bound in a complex with chaperones such as hsc70 for loading onto MHCII complexes found within target cells. Other studies have focused on the relative stimulatory capacity of mast cell exosomes, suggesting that there are clear differences between the responses of $\mathrm{T}$ and $\mathrm{B}$ cells based on the excitatory state of the mast cell during exosome release (Skokos et al., 2001a,b; de Vries et al., 2011).

We have addressed these questions using the RBL-2H3 cell line as a model system and confirmed that mast cells release exosomes through both basal (constitutive) secretion and regulated exocytosis (Figure 3, from Wilson Lab). Vesicles released by these two secretory pathways have distinct protein profiles (Figure 3A). As shown in Figure 3B (top), vesicles recovered from the supernatant of resting, cultured RBL cells are remarkably uniform, composed mostly of $100 \mathrm{~nm}$ exosomes with a dense core. In contrast, these distinctive vesicles (arrows in Figure 3B, bottom) represent only a subpopulation of the material released by activated cells. Insight into these distinct processes is provided by examination of the 


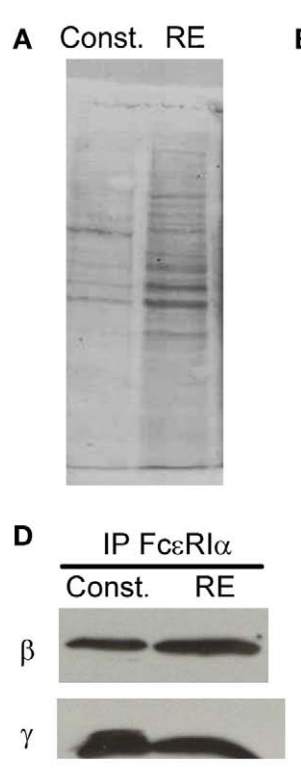

\section{B}
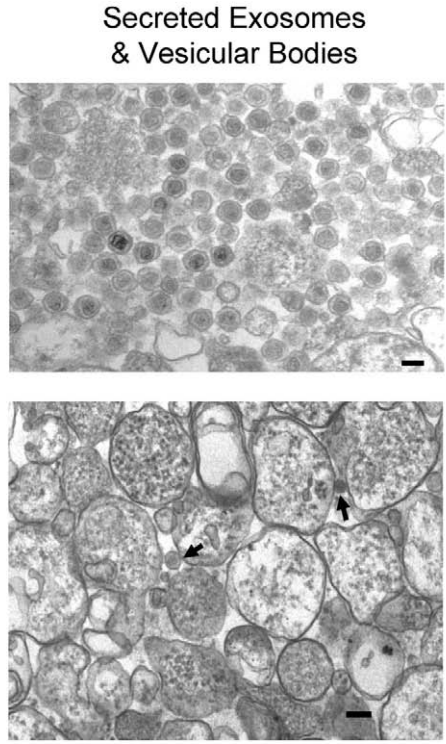

\section{Morphology of MVB} in live cells
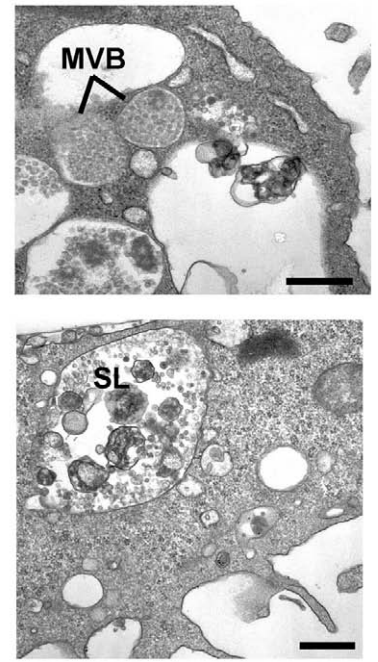

E
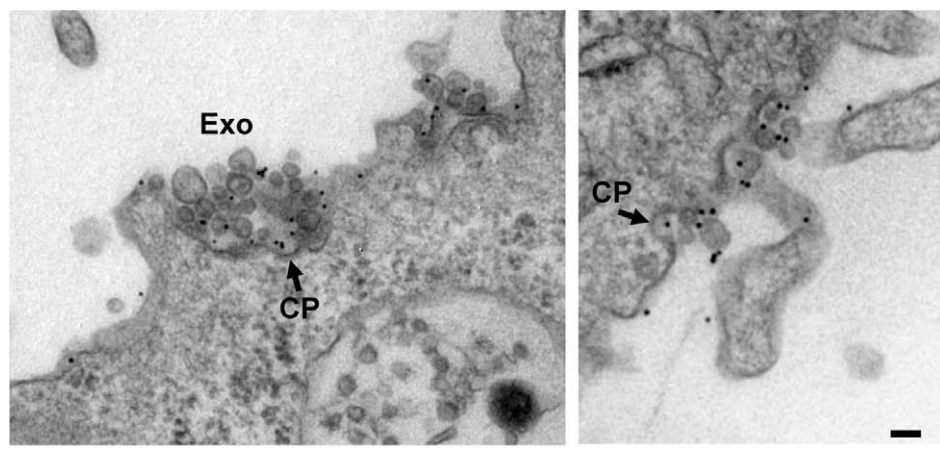

FIGURE 3 | RBL-2H3 exosomes. (A) Ponceau stain of protein profiles from exosomes released from MVBs constitutively (Const.) as compared to exosomes released from secretory lysosomes (RE) run on SDS-PAGE. (B) TEM of exosomes after secretion from cells that originated in MVBs (top left panel) or secretory lysosomes (bottom left panel, arrows; scale bars $=0.1 \mu \mathrm{m}$ ) and $\mathbf{( C )}$ exosomes still present in cells within MVBs (upper right panel) or secretory lysosomes (lower right panel, SL). Scale bars $=0.5 \mu \mathrm{m}$. (D) Blots demonstrating that exosomes contain intact FCERI. Immunoprecipitation of lysates with an $\alpha$-FceRI $\alpha$ antibody followed by blotting for $\beta$ and $\gamma$ demonstrate the subunits exist in a complexed state. (E) Exosomes with intact, IgE-bound receptors bind antigen and mediate reuptake of exosome-antigen complexes in coated pits. Representative TEM images of RBL-2H3 cells primed with $\alpha$-DNP IgE and stimulated with DNP-BSA-colloidal gold showing that gold-conjugated ligands target both the plasma membrane and exosome-associated FceRI. The presence of multiple exosomes within coated pits (denoted with arrows labeled $\mathrm{CP}$ ) shows reabsorption of exosomes with crosslinked receptor. Scale bars $=0.1 \mu \mathrm{m}$.
MVB of intact cells after fixation and preparation of ultra-thin sections for transmission electron microscopy (Figure 3C). In the top image of Figure 3C, lines point to two vesicles that are tightly packed with exosomes, suggesting these are the sources of constitutively secreted exosomes. In the lower image, "SL" marks a typical secretory lysosome that contains many convoluted vesicular structures as well as a number of exosomes. It is this compartment that fuses with the plasma membrane in response to antigen or calcium ionophore stimulation.

We further made the novel discovery that exosomes from both types of preparations contain intact FceRI, as demonstrated by immunoprecipitation of the FceRI $\alpha$ subunit and detection of both FceRI $\beta$ and $\gamma$ subunits by immunoblotting (Figure 3D). Importantly, electron microscopy experiments reveal that the exosomes are oriented "right-side out," exposing FceRI-IgE complexes and mediating their reuptake when crosslinked by polyvalent antigen (Figure 3E). For the mast cell, this represents a potential amplification mechanism where antigen is recycled back for continued crosslinking. In the context of exosome uptake by dendritic cells, B cells and other antigen presenting cells, we suggest that the delivery of mast cell-derived exosomes loaded with a cargo of $\operatorname{IgE}$ and antigen may promote robust presentation of allergenic peptides to T cells.

Finally, a novel role for mast cell exosomes may be the delivery of luminal contents to cells that engulf the vesicle. For example, mast cell exosomes can incorporate functional mRNA and microRNA into their lumens, which has been termed "exosomal shuttle RNA” or esRNA (Lotvall and Valadi, 2007; Eldh et al., 2010). This unique packaging mechanism can potentially initiate protein translation in the target cell using mRNA originally synthesized 
Mast Cell Intercellular Communication

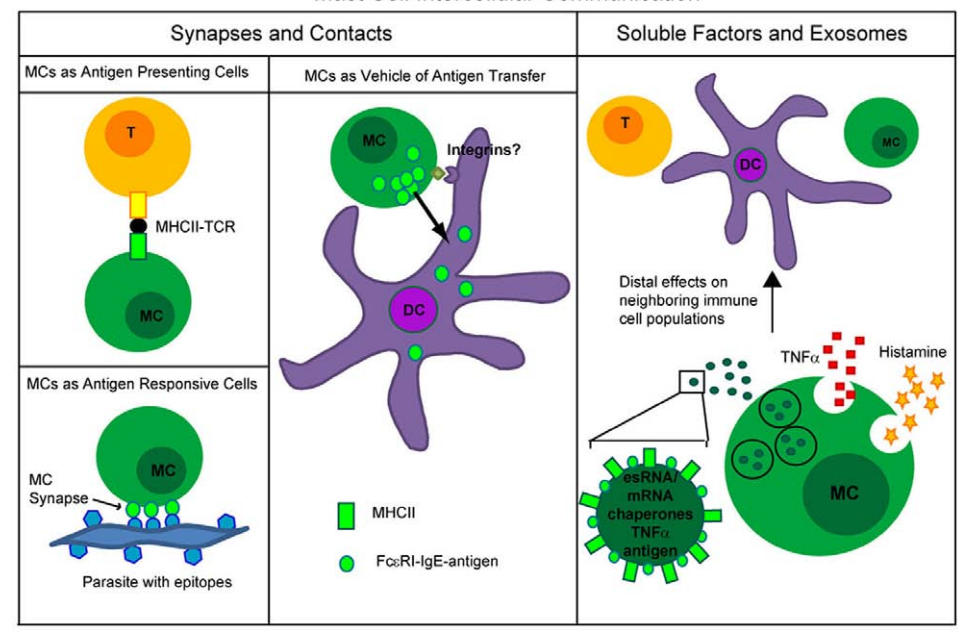

FIGURE 4 | Summary of types of mast cell intercellular communication occurring through formation of synapses or direct contacts versus through soluble factors or mast cell exosomes.

in the mast cells. It has been proposed that mast cells utilize this mechanism to broadcast local environmental signals to peripheral locations. Additionally, the packaging and transfer of esRNA between mast cells has been suggested as a mechanism for protection from harmful environmental effects such as oxidation or UV damage (Eldh et al., 2010).

\section{CONCLUSION}

The role for mast cells in modification of immune responses is extending beyond the scope of allergy alone. With capacity to secrete a variety of soluble mediators, form synapses, and deliver exosomes throughout the body, mast cells have several functional roles in immune modulation (Figure 4). Although this review focuses on the mechanism of mast cell modulation of the immune system, mast cells also serve as the target cells for modulation by other immune cells through soluble mediators, direct contact,

\section{REFERENCES}

Admyre, C., Johansson, S. M., Qazi, K. R., Filén, J. J., Lahesmaa, R., Norman, M., Neve, E. P., Scheynius, A., and Gabrielsson, S. (2007). Exosomes with immune modulatory features are present in human breast milk. J. Immunol. 179, 1969-1978.

Al-Nedawi, K., Szemraj, J., and Cierniewski, C. S. (2005). Mast cell-derived exosomes activate endothelial cells to secrete plasminogen activator inhibitor type 1 . Arterioscler. Thromb. Vasc. Biol. 25, 1744-1749.

Bacci, S., Pimpinelli, N., and Romagnoli, P. (2010). Contacts between mast cells and dendritic cells in the human skin. Ital. J. Anat. Embryol. 115, 25-30.

Bachelet, I., and Levi-Schaffer, F. (2007). Mast cells as effector cells:

or membrane vesicles/exosomes (Bhattacharyya et al., 1998; Shefler et al., 2010). Further elucidation of mast cell functions in the context of the tissue microenvironment will surely demonstrate the importance of this cell population as an emerging frontier in immunology.

\section{ACKNOWLEDGMENTS}

We thank Anna Holmes for performing immunohistochemistry. Fluorescent confocal and tissue images were generated in the University of New Mexico and Cancer Center Fluorescence Microscopy Shared Resource, funded as detailed on http://hsc.unm.edu/crtc/microscopy/Facility.html. Research presented was funded by the International Human Frontier Science Program Organization (RGY 0072/2008; awarded to Dr. Lidke and Dr. Cambi) and by the NIH grant R01 AI051575 (awarded to Dr. Wilson).

Buhner, S., and Schemann, M. (2012) Mast cell-nerve axis with a focus on the human gut. Biochim. Biophys. Acta 1822, 85-92.

Caby, M. P., Lankar, D., VincendeauScherrer, C., Raposo, G., and Bonnerot, C. (2005). Exosomal-like vesicles are present in human blood plasma. Int. Immunol. 17, 879-887.

Caron, G., Delneste, Y., Roelandts, E., Duez, C., Herbault, N., Magistrelli, G., Bonnefoy, J.Y., Pestel, J., and Jeannin, P. (2001). Histamine induces CD86 expression and chemokine production by human immature dendritic cells. J. Immunol. 166, 6000-6006.

Carrasco, Y. R., Fleire, S. J., Cameron, T., Dustin, M. L., and Batista, F. D. (2004). LFA-1/ICAM-1 interaction lowers the threshold of $\mathrm{B}$ cell activation by facilitating $\mathrm{B}$ cell adhesion and synapse formation. Immunity 20, 589-599.

Carroll-Portillo, A., Spendier, K., Pfeiffer, J., Griffiths, G., Li, H., Lidke, K. A., Oliver, J. M., Lidke, D. S., Thomas, J. L., Wilson, B. S., and Timlin, J. A. (2010). Formation of a mast cell synapse: Fc epsilon RI membrane dynamics upon binding mobile or immobilized ligands on surfaces. $J$. Immunol. 184, 1328-1338.

Caulfield, J. P., Hein, A., Moser, G., and Sher, A. (1981). Light and electron microscopic appearance of rat peritoneal mast cells adhering to schistosomula of Schistosoma mansoni by means of complement or antibody. J. Parasitol. 67, 776-783.

Cella, M., Sallusto, F., and Lanzavecchia, A. (1997). Origin, maturation and antigen presenting function of dendritic cells. Curr. Opin. Immunol. 9, 10-16. 
Dawicki, W., Jawdat, D. W., Xu, N., and Marshall, J. S. (2010). Mast cells, histamine, and IL-6 regulate the selective influx of dendritic cell subsets into an inflamed lymph node. $J$. Immunol. 184, 2116-2123.

de Vries, V. C., Pino-Lagos, K., Nowak, E. C., Bennett, K. A., Oliva, C., and Noelle, R. J. (2011). Mast cells condition dendritic cells to mediate allograft tolerance. Immunity 35 , 550-561.

Denzer, K., Kleijmeer, M. J., Heijnen, H. F., Stoorvogel, W., and Geuze, H. J. (2000). Exosome: from internal vesicle of the multivesicular body to intercellular signaling device. J. Cell. Sci. 113(Pt 19), 3365-3374.

Dudeck, A., Suender, C. A., Kostka, S. L., von Stebut, E., and Maurer, M. (2011). Mast cells promote Th1 and Th17 responses by modulating dendritic cell maturation and function. Eur. J. Immunol. 41, 1883-1893.

Dustin, M. L. (2008). T-cell activation through immunological synapses and kinapses. Immunol. Rev. 221, 77-89.

Dustin, M. L., Chakraborty, A. K., and Shaw, A. S. (2010). Understanding the structure and function of the immunological synapse. Cold Spring Harb. Perspect. Biol. 2, a002311.

Dustin, M. L., Miller, J. M., Ranganath, S., Vignali, D. A., Viner, N. J., Nelson, C. A., and Unanue, E. R. (1996). TCR-mediated adhesion of $\mathrm{T}$ cell hybridomas to planar bilayers containing purified MHC class II/peptide complexes and receptor shedding during detachment. J. Immunol. 157, 2014-2021.

Eldh, M., Ekström, K., Valadi, H., Sjöstrand, M., Olsson, B., Jernås, M., and Lötvall, J. (2010). Exosomes communicate protective messages during oxidative stress; possible role of exosomal shuttle RNA. PLoS ONE 5, e15353. doi:10.1371/journal.pone. 0015353

Galli, S. J., and Wershil, B. K. (1995). Mouse mast cell cytokine production: role in cutaneous inflammatory and immunological responses. Exp. Dermatol. 4(Pt 2), 240-249.

Gaudenzio, N., Espagnolle, N., Mars, L. T., Liblau, R., Valitutti, S., and Espinosa, E. (2009). Cell-cell cooperation at the $\mathrm{T}$ helper cell/mast cell immunological synapse. Blood 114, 4979-4988.

Grabbe, J., Karau, L., Welker, P., Ziegler, A., and Henz, B. M. (1997). Induction of MHC class II antigen expression on human HMC-1 mast cells. J. Dermatol. Sci. 16, 67-73.
Gurish, M. F., and Austen, K. F. (2001). The diverse roles of mast cells. J. Exp. Med. 194, F1-F5.

Hershko, A. Y., and Rivera, J. (2010). Mast cell and $\mathrm{T}$ cell communication; amplification and control of adaptive immunity. Immunol. Lett. 128, 98-104.

Inamura, N., Mekori, Y. A., Bhattacharyya, S. P., Bianchine, P. J., and Metcalfe, D. D. (1998). Induction and enhancement of Fc(epsilon)RI-dependent mast cell degranulation following coculture with activated $\mathrm{T}$ cells: dependency on ICAM-1- and leukocyte function-associated antigen (LFA)1-mediated heterotypic aggregation. J. Immunol. 160, 4026-4033.

Kaplan, D. H. (2010). In vivo function of Langerhans cells and dermal dendritic cells. Trends Immunol. 31, 446-451.

Ketavarapu, J. M., Rodriguez, A. R., Yu, J. J., Cong, Y., Murthy, A. K., Forsthuber, T. G., Guentzel, M. N., Klose, K. E., Berton, M. T., and Arulanandam, B. P. (2008). Mast cells inhibit intramacrophage Francisella tularensis replication via contact and secreted products including IL-4. Proc. Natl. Acad. Sci. U.S.A. 105, 9313-9318.

Kitawaki, T., Kadowaki, N., Sugimoto, N., Kambe, N., Hori, T., Miyachi, Y., Nakahata, T., and Uchiyama, T. (2006). IgE-activated mast cells in combination with pro-inflammatory factors induce Th2-promoting dendritic cells. Int. Immunol. 18, 1789-1799.

Kunder, C. A., St. John, A. L., Li, G., Leong, K. W., Berwin, B., Staats, H F., and Abraham, S. N. (2009). Mast cell-derived particles deliver peripheral signals to remote lymph nodes. J. Exp. Med. 206, 2455-2467.

Kushwah, R., and Hu, J. (2011). Complexity of dendritic cell subsets and their function in the host immune system. Immunology 133 409-419.

Laulagnier, K., Hamdi, S., Subra, C., Lankar, D., and Record, M. (2005). Characterization of exosome subpopulations from RBL-2H3 cells using fluorescent lipids. Blood Cells Mol. Dis. 35, 116-121.

Laulagnier, K., Motta, C., Hamdi, S., Roy, S., Fauvelle, F., Pageaux, J. F., Kobayashi, T., Salles, J. P., Perret, B., Bonnerot, C., and Record, M. (2004). Mast cell- and dendritic cell-derived exosomes display a specific lipid composition and an unusual membrane organization. Biochem. J. 380(Pt 1), 161-171.
Lin, K. B., Freeman, S. A., Zabetian, S., Brugger, H., Weber, M., Lei, V. Dang-Lawson, M., Tse, K. W., Santamaria, R., Batista, F. D., and Gold, M. R. (2008). The rap GTPases regulate $B$ cell morphology, immune-synapse formation, and signaling by particulate $\mathrm{B}$ cell receptor ligands. Immunity 28, 75-87.

Lotvall, J., and Valadi, H. (2007). Cell to cell signalling via exosomes through esRNA. Cell Adh. Migr. 1, 156-158.

Marshall, J. S. (2004). Mast-cell responses to pathogens. Nat. Rev. Immunol. 4, 787-799.

Matsushima, H., Yamada, N., Matsue, H., and Shimada, S. (2004). TLR3-, TLR7-, and TLR9-mediated production of proinflammatory cytokines and chemokines from murine connective tissue type skin-derived mast cells but not from bone marrowderived mast cells. J. Immunol. 173, 531-541.

Mekori, Y. A., and Baram, D. (2002). Heterotypic adhesion-induced mast cell activation: biologic relevance in the inflammatory context. Mol. Immunol. 38, 1363-1367.

Merluzzi, S., Frossi, B., Gri, G., Parusso, S., Tripodo, C., and Pucillo, C. (2010). Mast cells enhance proliferation of $\mathrm{B}$ lymphocytes and drive their differentiation toward IgAsecreting plasma cells. Blood 115, 2810-2817.

Otsuka, A., Kubo, M., Honda, T., Egawa, G., Nakajima, S., Tanizaki, H., Kim, B., Matsuoka, S., Watanabe, T., Nakae, S., Miyachi, Y., and Kabashima, K. (2011). Requirement of interaction between mast cells and skin dendritic cells to establish contact hypersensitivity. PLoS ONE 6, e25538. doi:10.1371/journal.pone.0025538

Palanisamy, V., Sharma, S., Deshpande, A., Zhou, H., Gimzewski, J., and Wong, D. T. (2010). Nanostructural and transcriptomic analyses of human saliva derived exosomes. PLoS ONE 5, e8577. doi:10.1371/journal.pone.0008577

Pfeiffer, J. R., Seagrave, J. C., Davis, B. H., Deanin, G. G., and Oliver, J. M. (1985). Membrane and cytoskeletal changes associated with IgEmediated serotonin release from rat basophilic leukemia cells. J. Cell Biol. 101, 2145-2155.

Pisitkun, T., Shen, R. F., and Knepper, M. A. (2004). Identification and proteomic profiling of exosomes in human urine. Proc. Natl. Acad. Sci. U.S.A. 101, 13368-13373.

Raposo, G., Tenza, D., Mecheri, S. Peronet, R., Bonnerot, C., and
Desaymard, C. (1997). Accumulation of major histocompatibility complex class II molecules in mast cell secretory granules and their release upon degranulation. Mol. Biol. Cell 8, 2631-2645.

Ren, S. R., Xu, L. B., Wu, Z. Y., Du, J., Gao, M. H., and Qu, C. F. (2010). Exogenous dendritic cell homing to draining lymph nodes can be boosted by mast cell degranulation. Cell. Immunol. 263, 204-211.

Ribatti, D., and Crivellato, E. (2011). Mast cells, angiogenesis and cancer. Adv. Exp. Med. Biol. 716, 270-288.

Romani, N., Clausen, B. E., and Stoitzner, P. (2010). Langerhans cells and more: langerinexpressing dendritic cell subsets in the skin. Immunol. Rev. 234, 120-141.

Saha, B., Tonkal, A. M., Croft, S., and Roy, S. (2004). Mast cells at the hostpathogen interface: host-protection versus immune evasion in leishmaniasis. Clin. Exp. Immunol. 137, 19-23.

Shefler, I., Salamon, P., Reshef, T., Mor, A., and Mekori, Y. A. (2010). T cell-induced mast cell activation: a role for microparticles released from activated T cells. J. Immunol. 185, 4206-4212.

Shelburne, C. P., Nakano, H., St. John, A. L., Chan, C., McLachlan, J. B., Gunn, M. D., Staats, H. F., and Abraham, S. N. (2009). Mast cells augment adaptive immunity by orchestrating dendritic cell trafficking through infected tissues. Cell Host Microbe 6, 331-342.

Skokos, D., Botros, H. G., Demeure, C., Morin, J., Peronet, R., Birkenmeier, G., Boudaly, S., and Mécheri S. (2003). Mast cell-derived exosomes induce phenotypic and functional maturation of dendritic cells and elicit specific immune responses in vivo. J. Immunol. 170, 3037-3045.

Skokos, D., Le Panse, S., Villa, I., Rousselle, J. C., Peronet, R., David, B., Namane, A., and Mécheri, S. (2001a). Mast cell-dependent B and T lymphocyte activation is mediated by the secretion of immunologically active exosomes. J. Immunol. 166, 868-876.

Skokos, D., Le Panse, S., Villa, I., Rousselle, J. C., Peronet, R., Namane, A., David, B., and Mécheri, S. (2001b). Nonspecific B and T cell-stimulatory activity mediated by mast cells is associated with exosomes. Int. Arch. Allergy Immunol. 124 133-136. 
Soloff, A. C., and Barratt-Boyes, S. M. (2010). Enemy at the gates: dendritic cells and immunity to mucosal pathogens. Cell Res. 20, 872-885.

Stoorvogel, W., Kleijmeer, M. J., Geuze, H. J., and Raposo, G. (2002). The biogenesis and functions of exosomes. Traffic 3, 321-330.

Supajatura, V., Ushio, H., Nakao, A., Okumura, K., Ra, C., and Ogawa, H. (2001). Protective roles of mast cells against enterobacterial infection are mediated by Toll-like receptor 4 . J. Immunol. 167, 2250-2256.

Tamir, I., and Cambier, J. C. (1998). Antigen receptor signaling: integration of protein tyrosine kinase functions. Oncogene 17, Wesolowski, J., and Paumet, F. (2011). 1353-1364.

Thery, C., Ostrowski, M., and Segura, E. (2009). Membrane vesicles as conveyors of immune responses. Nat. Rev. Immunol. 9, 581-593.

Valitutti, S., and Espinosa, E. (2010). Cognate interactions between mast cells and helper T lymphocytes. Self Nonself 1, 114-122.

Vincent-Schneider, H., Théry, C. Mazzeo, D., Tenza, D., Raposo, G., and Bonnerot, C. (2001). Secretory granules of mast cells accumulate mature and immature MHC class II molecules. J. Cell. Sci. 114(Pt 2), 323-334.
The impact of bacterial infection on mast cell degranulation. Immunol. Res. 51, 215-226.

Conflict of Interest Statement: The authors declare that the research was conducted in the absence of any commercial or financial relationships that could be construed as a potential conflict of interest.

Received: 19 December 2011; accepted: 27 February 2012; published online: 15 March 2012.

Citation: Carroll-Portillo A, Surviladze $Z$, Cambi A, Lidke DS and Wilson BS
(2012) Mast cell synapses and exosomes: membrane contacts for information exchange. Front. Immun. 3:46. doi: 10.3389/fimmu.2012.00046

This article was submitted to Frontiers in Inflammation, a specialty of Frontiers in Immunology.

Copyright (C) 2012 Carroll-Portillo, Surviladze, Cambi, Lidke and Wilson. This is an open-access article distributed under the terms of the Creative Commons Attribution Non Commercial License, which permits non-commercial use, distribution, and reproduction in other forums, provided the original authors and source are credited. 